\title{
Electrostatic Potentials and Their Spatial Derivatives About Point Defects in Ionic Crystals
}

\author{
Herbert S. Bennett \\ Institute for Materials Research, National Bureau of Standards, Washington, D.C. 20234
}

(June 10, 1968)

\begin{abstract}
The electrostatic potential which arises from a lattice array of point ions is computed in terms of a Taylor's series expansion for small distances from a lattice site. This expansion gives the change in electrostatic energy when an ion moves in the background of a perfect point ion lattice potential. The Taylor's series coefficients for terms up to fourth order in the ion displacement are evaluated for the $\mathrm{NaCl}$ and $\mathrm{CaF}_{2}$ lattice structures.
\end{abstract}

Key Words: $\mathrm{CaF}_{2}$; classical ionic lattice theory; electrostatic potential; lattice distortion; NaCl; point defect.

\section{Introduction}

Most theoretical studies of defects in ionic solids require a knowledge of the manner in which the lattice distorts to accommodate the defect. Classical ionic lattice theory gives the interaction energy $U_{\mu \nu}$ between two ions $\mu$ and $\nu$ at a separation $r_{\mu \nu}=\left|\mathbf{r}_{\mu}-\mathbf{r}_{\nu}\right|$; namely,

$$
\begin{aligned}
U_{\mu \nu}=\left(Z_{\mu} Z_{\nu} / r_{\mu \nu}\right)-\left(C_{\mu \nu}^{(6)} / r_{\mu \nu}^{6}\right) & \\
& -\left(C_{\mu \nu}^{(8)} / r_{\mu \nu}^{8}\right)+\varphi_{\text {rep }}\left(r_{\mu \nu}\right),
\end{aligned}
$$

where the four terms are respectively the coulomb electrostatic, dipole-dipole, dipole-quadrupole, and repulsive contributions to the lattice energy. The charge on the ion $\nu$ is $Z_{\nu}, C_{\mu \nu}^{(6)}$ and $C_{\mu \nu}^{(8)}$ are the van der Waals constants for ions $\mu$ and $\nu$, and the repulsive energy $\varphi_{\text {rep }}\left(r_{\mu \nu}\right)$ takes the Pauli exclusion principle between the $\mu$ th and the $\nu$ th ion cores into account. The repulsive energy $\varphi_{\text {rep }}$ is a short-range function of $r_{\mu \nu}$. The cohesive energy $\Phi\left(r_{0}\right)$ for the crystal becomes,

$$
\Phi\left(r_{0}\right)=\frac{1}{2} \sum_{\mu \neq \nu} U_{\mu \nu}+U_{0},
$$

where $r_{0}$ is the nearest neighbor distance for the perfect lattice and where $U_{0}$ is the lattice energy.

The presence of a defect causes the neighboring ions to move from their perfect lattice sites. This motion will modify the energy of the lattice and this change in the lattice energy is most important in a study of the properties of defects in ionic solids. Among the many terms which occur in the change in the lattice energy due to a defect is the one which represents the change in electrostatic energy when a neighboring ion moves in the background of a perfect point ion lattice potential.

As an example, let us consider the $F$ center, which is an electron localized about an anion vacancy. The ions neighboring the $F$ center defect move in a self consistent manner to accommodate the $F$ electron. The many terms in the change in lattice energy are grouped in some convenient manner. One of these terms is the change in electrostatic energy $\Delta V_{\nu}$ which occurs when a neighboring cation moves in the background of a perfect point ion lattice potential [1];1

$$
\Delta V_{\nu}\left(\mathbf{r}_{\nu}^{\prime}-\mathbf{r}_{\nu}\right)=Z_{\nu} \sum_{\mu \neq \nu} z_{\mu}\left\{\left|\mathbf{r}_{\nu}^{\prime}-\mathbf{r}_{\mu}\right|^{-1}-\left|\mathbf{r}_{\nu}-\mathbf{r}_{\mu}\right|^{-1}\right\}
$$

The quantity $\Delta V_{\nu}\left(\mathbf{r}_{\nu}^{\prime}-\mathbf{r}_{v}\right)$ is the change in electrostatic coulombic energy when ion $\nu$ moves from the perfect lattice site $\mathbf{r}_{\nu}$ to the position $\mathbf{r}_{\nu}^{\prime}$, which is not a perfect lattice site, in the background of a perfect lattice point ion potential. Denoting the distortion (ion displacement) by $\mathbf{r}=\mathbf{r}_{\nu}^{\prime}-\mathbf{r}_{\nu}$ we write the energy of the $\nu$ th ion at the position $\mathbf{r}_{\nu}^{\prime}=\mathbf{r}_{\nu}+\mathbf{r}$ in the form,

$$
V_{\nu}(\mathbf{r})=Z_{\nu} \sum_{\mu \neq \nu} Z_{\mu}\left|\mathbf{r}_{\nu}-\mathbf{r}_{\mu}+\mathbf{r}\right|^{-1} .
$$

\footnotetext{
${ }^{1}$ Figures in brackets indicate the literature references at the end of this paper.
} 
Hence, the change in the electrostatic coulomb energy when only the $\nu$ th ion moves in the background of a perfect lattice is

$$
\Delta V_{\nu}(\mathbf{r})=V_{\nu}(\mathbf{r})-V_{\nu}(\mathbf{r}=0) .
$$

The few researchers $[2,3,4]$, who have considered local lattice distortions near the $F$ center in a manner self consistent with the $F$ electron state, consider only the alkali halides and neglect this term $\Delta V_{\nu}$. This may be reasonable in the alkali halides for optical absorption discussions in which the lattice distortions are small. But one may question their neglecting the term $\Delta V_{\nu}$ for optical emission studies in the alkali halides and for other optical discussions in the alkaline earth fluorides and oxides. The lattice distortion may be as large as twelve percent of the nearest neighbor distance $r_{0}$ for the relaxed excited state in the alkali halides and in the alkaline earth halides and for all states in the alkaline earth oxides. In this paper we shall express the term $\Delta V_{\nu}$ as a series expansion in the distortion $\mathbf{r}=\mathbf{r}_{\nu}^{\prime}-\mathbf{r}_{\nu}$ and evaluate by Ewald's method [5] the lattice summations which give the series coefficients. We shall compute explicitly these coefficients for the $\mathrm{NaCl}$ and $\mathrm{CaF}_{2}$ lattice structures.

\section{Formulation}

When $\left(r / r_{0}\right)<1$, we may expand the electrostatic energy given by eq (4) in a Taylor's series about the point $\mathbf{r}=\mathbf{0}$;

$$
\begin{gathered}
V_{\nu}(\mathbf{r}) \approx V_{\nu}(\mathbf{r}=0)+\mathbf{r} \cdot \boldsymbol{\nabla} V_{\nu}(\mathbf{r})+\frac{1}{2}(\mathbf{r} \cdot \boldsymbol{\nabla})^{2} V_{\nu}(\mathbf{r}) \\
+\frac{1}{6}(\mathbf{r} \cdot \boldsymbol{\nabla})^{3} V_{\nu}(\mathbf{r})+\frac{1}{24}(\mathbf{r} \cdot \boldsymbol{\nabla})^{4} V_{\nu}(\mathbf{r})+\ldots .
\end{gathered}
$$

The various derivatives of $V_{\nu}(\mathbf{r})$ with respect to the Cartesian components of $\mathbf{r}$ are evaluated at $\mathbf{r}=\mathbf{0}$. Many of the derivatives in eq (6) will be zero by symmetry arguments, but which ones are zero will depend upon the specific lattice structure and upon whether an anion or a cation moves.

Let us first consider the point lattices and the reciprocal lattices for the $\mathrm{NaCl}$ and $\mathrm{CaF}_{2}$ structures. The primitive translational vectors for these two lattices are

$$
\mathbf{a}_{1}=(a / 2)(0,1,1), \quad \mathbf{a}_{2}=(a / 2)(1,0,1)
$$

and $\quad \mathbf{a}_{3}=(a / 2)(1,1,0)$

and the volume of the unit cell $v_{c}$ is

$$
v_{c}=\mathbf{a}_{1} \cdot\left(\mathbf{a}_{2} \times \mathbf{a}_{3}\right)=\left(a^{3} / 4\right) .
$$

The lattice constant $a$ is the $\mathrm{Na}-\mathrm{Na}$ distance or the
$\mathrm{Ca}-\mathrm{Ca}$ distance. When a cation is at the origin, the position vector $\mathbf{r}_{l}^{+}$for the other cations $\left(\mathrm{Na}^{+}\right.$or $\left.\mathrm{Ca}^{++}\right)$is

$$
\mathbf{r}_{l}^{+}=(a / 2)\left[\left(l_{2}+l_{3}\right) \hat{x}+\left(l_{3}+l_{1}\right) \hat{y}+\cdot\left(l_{1}+l_{2}\right) \hat{z}\right]
$$

where the $l_{i}$ 's are $0, \pm 1, \pm 2, \pm 3$, etc. The anions for $\mathrm{NaCl}$ structures are located at the sites

$$
\mathbf{r}_{l}^{-}=\mathbf{r}_{l}^{+}+\mathbf{x}_{a},
$$

where $\mathbf{x}_{a}=(a / 2)(\hat{x}+\hat{y}+\hat{z})$ and the anions for the $\mathrm{CaF}_{2}$ structures are located at the sites

$$
\mathbf{r}_{l}^{-}(1)=\mathbf{r}_{l}^{+}+\mathbf{x}_{a}(1) \text { and } \mathbf{r}_{l}^{-}(2)=\mathbf{r}_{l}^{+}+\mathbf{x}_{a}(2),
$$

where

$$
\mathbf{x}_{a}(1)=(a / 4)(\hat{x}+\hat{y}+\hat{z}) \text { and } \mathbf{x}_{a}(2)=(3 a / 4)(\hat{x}+\hat{y}+\hat{z}) \text {. }
$$

We also choose the distortion $\mathbf{r}$ to be given by

$$
\mathbf{r}=(a / 4)\left(\sigma_{1} \hat{x}+\sigma_{2} \hat{y}+\sigma_{3} \hat{z}\right) .
$$

The position of a nearest neighbor cation when an anion is the reference ion is $\mathbf{r}_{c}=(a / 2)(0,0,1)$ for $\mathrm{NaCl}$ structures and $\mathbf{r}_{c}=(a / 4)(1,1,1)$ for $\mathrm{CaF}_{2}$ structures. The nearest neighbor distance $r_{0}$ for the perfect lattice is then $r_{0}=(a / 2)$ for $\mathrm{NaCl}$ structures and $r_{0}=(\sqrt{3} a / 4)$ for $\mathrm{CaF}_{2}$ structures. Thus,

$$
\nu_{c}(\mathrm{NaCl})=2 r_{0}^{3} \text { and } \nu_{c}\left(\mathrm{CaF}_{2}\right)=(16 / 3 \sqrt{3}) r_{0}^{3} .
$$

The first term of eq (6) is directly proportional to the Madelung constant for the $\nu$ th ion, $\alpha_{\nu}=\left[V_{\nu}(\mathbf{r}=\mathbf{0}) r_{0} / Z_{\nu}\right]$. Throughout this paper, we will use the nearest neighbor distance as the reference distance.

The vectors $\mathbf{b}_{1}=(1 / a)(-1,1,1), \mathbf{b}_{2}=(1 / a)(1,-1,1)$ and $\mathbf{b}_{3}=(1 / a)(1,1,-1)$ are the reciprocal triad of the primitive translational vectors $\mathbf{a}_{1}, \mathbf{a}_{2}$, and $\mathbf{a}_{3}$. The wave vector in the reciprocal lattice is

$$
\mathbf{g}=2 \pi\left(n_{1} \mathbf{b}_{1}+n_{2} \mathbf{b}_{2}+n_{3} \mathbf{b}_{3}\right)
$$

where the $n_{i}$ 's are $0, \pm 1, \pm 2, \pm 3$, etc.

The series given in eq (4) is conditionally convergent. Hence, straightforward evaluations for such series will be most tedious and usually unsatisfactory. The Ewald's method [5, 6] is an elegant procedure by which one converts the series (4) into the sum of two series-each one of which converges rapidly. Referring the reader to references five and six for the details of Ewald's method, we have the following representation for the lattice summations appearing in eq (4):

$$
\begin{aligned}
\varphi(\mathbf{x} ; \mathbf{r}) & =\sum_{1}\left|\mathbf{r}_{l}^{+}+\mathbf{x}+\mathbf{r}\right|^{-1} \\
& =\frac{\pi}{v_{c}} \frac{1}{G^{2}} \sum_{\mathbf{g}} \frac{\exp \left(-g^{2} / 4 G^{2}\right)}{\left(g^{2} / 4 G^{2}\right)} \cos \mathbf{g} \cdot(\mathbf{x}+\mathbf{r}) \\
& +\sum_{1}\left|\mathbf{r}_{l}^{+}+\mathbf{x}+\mathbf{r}\right|^{-1} \operatorname{erfc}\left\{G\left|\mathbf{r}_{l}^{+}+\mathbf{x}+\mathbf{r}\right|\right\},
\end{aligned}
$$


when

$$
\mathbf{x}+\mathbf{r} \neq 0
$$

and

$$
\begin{aligned}
\varphi(\mathbf{x}=0 ; \mathbf{r}=0)= & \sum_{1 \neq 0}\left|\mathbf{r}_{l}^{+}\right|^{-1} \\
= & \frac{\pi}{v_{c}} \frac{1}{G^{2}} \sum \frac{\exp \left(-g^{2} / 4 G^{2}\right)}{\left(g^{2} / 4 G^{2}\right)} \\
& +\sum_{l \neq 0}\left|\mathbf{r}_{l}^{+}\right|^{-1} \operatorname{erfc}\left\{G\left|\mathbf{r}_{l}^{+}\right|\right\}-\frac{2 G}{\sqrt{\pi}} .
\end{aligned}
$$

We choose the quantity $G$ so that the series in $\mathbf{g}$ and the series in $\mathbf{I}$ are both rapidly convergent. The complementary error function erfc $(z)$ is defined by

$$
\operatorname{erfc}(z)=(2 / \sqrt{\pi}) \int_{z}^{\infty} \exp \left(-y^{2}\right) d y
$$

and is normalized so that erfe $(0)=1$. Hence, the potential energy for a cation displaced a distance $\mathbf{r}$ from its perfect lattice site is

$$
V_{c}(\mathrm{NaCl} ; \mathbf{r})=Z_{c}\left[Z_{c} \varphi(\mathbf{x}=0 ; \mathbf{r})+Z_{a} \varphi\left(\mathbf{x}_{a} ; \mathbf{r}\right)\right],
$$

and

$$
\begin{aligned}
V_{c}\left(\mathrm{CaF}_{2} ; \mathbf{r}\right)=Z_{c}\left[Z_{c} \varphi(\mathbf{x}=0 ; \mathbf{r})\right. & +Z_{a}\left\{\varphi\left(\mathbf{x}_{a}(1) ; \mathbf{r}\right)\right. \\
& \left.\left.+\varphi\left(\mathbf{x}_{a}(2) ; \mathbf{r}\right)\right\}\right] ;
\end{aligned}
$$

and the potential energy for an anion displaced a distance $\mathbf{r}$ from its perfect lattice site is

$$
\begin{aligned}
V_{a}(\mathrm{NaCl} ; \mathbf{r})=Z_{a}\left[Z_{c} \varphi(\mathbf{x}=\right. & \left.\mathbf{0} ; \mathbf{r}-\mathbf{x}_{a}\right) \\
& \left.+Z_{a} \varphi\left(\mathbf{x}_{a} ; \mathbf{r}-\mathbf{x}_{a}\right)\right],
\end{aligned}
$$

and

$V_{a}\left(\mathrm{CaF}_{2} ; \mathbf{r}\right)=Z_{a}\left[Z_{c} \varphi\left(\mathbf{x}=\mathbf{0} ; \mathbf{r}-\mathbf{x}_{a}(1)\right)+Z_{a}\left\{\varphi\left(\mathbf{x}_{a}(1) ;\right.\right.\right.$

$$
\left.\left.\left.\mathbf{r}-\mathbf{x}_{a}(1)\right)+\varphi\left(\mathbf{x}_{a}(2) ; \mathbf{r}-\mathbf{x}_{a}(2)\right)\right\}\right] .
$$

The ionicity of the cations is $Z_{c}$ and the ionicity of the anions is $Z_{a}$.

We then see from expansion (6) that the term $V_{\nu}(\mathbf{r}=\mathbf{0})$ and the following types of derivatives

$$
\left.\left(\partial^{\prime \prime} V_{\nu}(\mathbf{r}) / \partial x^{s} \partial y^{\prime} \partial z^{u}\right)\right|_{\mathbf{r}=0}
$$

must be evaluated, where $n, s, t$, and $u$ are zero and positive integers and where $n=s+t+u$. We shall use the Ewald representation to compute the term $V_{\nu}(\mathbf{r}=\mathbf{0})$ in expansion (6). Because the spatial derivatives of the lattice summation (4) for $V_{\nu}(\mathbf{r})$ lead to expressions which converge more rapidly than the lattice summation (4) itself converges, we find that Ewald's method is not necessary whenever we evaluate $\left(\partial^{\prime \prime} V_{\nu}(\mathbf{r}) / \partial x^{s} \partial y^{t} \partial z^{u}\right)$ for $n \geqslant 4$. The evaluation of such derivatives is straight forward but is very tedious. We tabulate in appendix A these derivatives up to order four; i.e., $n \leqslant 4$.

\section{Results}

Combining eqs (6), (7) and the equations in appendix $A$, we write the electrostatic energy in the form,

$$
\begin{aligned}
& V_{\nu}(\mathbf{r})=\frac{\alpha_{\nu}}{r_{0}}+\frac{1}{r_{0}}\left\{\left(\sigma_{1}+\sigma_{2}+\sigma_{3}\right) V_{\nu x}\right. \\
& +\frac{1}{2}\left(\sigma_{1}^{2}+\sigma_{2}^{2}+\sigma_{3}\right) V_{\nu 2 x}+\left(\sigma_{1} \sigma_{2}+\sigma_{2} \sigma_{3}+\sigma_{3} \sigma_{1}\right) V_{\nu x y} \\
& +\frac{1}{6}\left(\sigma_{1}^{3}+\sigma_{2}^{3}+\sigma_{3}^{3}\right) V_{\nu 3 x} \\
& +\frac{1}{2}\left(\sigma_{1}^{2} \sigma_{2}+\sigma_{2}^{2} \sigma_{3}+\sigma_{3}^{2} \sigma_{1}+\sigma_{1} \sigma_{2}^{2}+\sigma_{2} \sigma_{3}^{2}+\sigma_{3} \sigma_{1}^{2}\right) V_{\nu 2 x y} \\
& +\sigma_{1} \sigma_{2} \sigma_{3} V_{\nu x y z}+\frac{1}{24}\left(\sigma_{1}^{4}+\sigma_{2}^{4}+\sigma_{3}^{4}\right) V_{\nu 4 x} \\
& +\frac{1}{6}\left(\sigma_{1} \sigma_{2}^{3}+\sigma_{1}^{3} \sigma_{2}+\sigma_{2} \sigma_{3}^{3}+\sigma_{2}^{3} \sigma_{3}+\sigma_{3} \sigma_{1}^{3}+\sigma_{3}^{1} \sigma_{1}\right) V_{\nu 3 x y} \\
& +\frac{1}{4}\left(\sigma_{1}^{2} \sigma_{2}^{2}+\sigma_{2}^{2} \sigma_{3}^{2}+\sigma_{3}^{2} \sigma_{1}^{2}\right) V_{\nu 2 x 2 y}
\end{aligned}
$$

\begin{tabular}{|c|c|c|c|c|}
\hline \multirow{2}{*}{ Reference ion } & \multicolumn{2}{|c|}{$\mathrm{NaCl}$ structures } & \multicolumn{2}{|c|}{$\mathrm{CaF}_{2}$ structures } \\
\hline & Cation & Anion & Cation & Anion \\
\hline 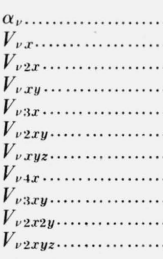 & $\begin{array}{l}-1.7476 \\
0.0 \\
0.0 \\
0.0 \\
0.0 \\
0.0 \\
0.0 \\
-.2148+02 \\
0.0 \\
+.1074+02 \\
0.0\end{array}$ & $\begin{array}{c}+1.7476 \\
0.0 \\
0.0 \\
0.0 \\
0.0 \\
0.0 \\
0.0 \\
+.2148+02 \\
0.0 \\
-.1074+02 \\
0.0\end{array}$ & $\begin{array}{l}-7.565 \\
0.0 \\
0.0 \\
0.0 \\
0.0 \\
0.0 \\
0.0 \\
+.2983+02 \\
0.0 \\
-.1491+02 \\
0.0\end{array}$ & $\begin{array}{l}+4.070 \\
0.0 \\
0.0 \\
0.0 \\
0.0 \\
0.0 \\
.1664+02 \\
-.4843+02 \\
0.0 \\
+.2423+02 \\
0.0\end{array}$ \\
\hline
\end{tabular}$$
\left.+\frac{1}{2}\left(\sigma_{1}^{2} \sigma_{2} \sigma_{3}+\sigma_{1} \sigma_{2}^{2} \sigma_{3}+\sigma_{1} \sigma_{2} \sigma_{3}^{2}\right) V_{\nu 2 x y z}+\cdots \cdot\right\} .
$$

The series coefficients $V_{\nu s x t y u z}$ are the derivatives $\left(\partial^{\prime \prime} V_{\nu} / \partial x^{s} \partial y^{t} \partial z^{u}\right)$ evaluated at $\mathbf{r}=\mathbf{0}$. We list in table 1 the Madelung constants $\alpha_{\nu}$ and the series coefficients $V_{\nu \text { sxtyuz }}$ for the $\mathrm{NaCl}$ and $\mathrm{CaF}_{2}$ structures.

TABLE 1. The Madelung constant $\alpha_{\nu}$ and the series coefficients

$\mathrm{V}_{\nu_{\text {sxtyuz }}}$ which appear in eq (14) for $\mathrm{NaCl}$ and $\mathrm{CaF}_{2}$ structures

The reference ion moves a distance $\mathbf{r}$ given by eq (7) from its perfect lattice site. The reference distance is the nearest neighbor distance $r_{0} ; r_{0}=(a / 2)$ for $\mathrm{NaCl}$ structures and $r_{0}=(\sqrt{3} a / 4)$ for $\mathrm{CaF}_{2}$ structures. The number $N \times 10^{+n}$ is denoted by $N+0 n$. 
The author acknowledges several helpful conversations with A. D. Franklin.

\section{Appendix A: Derivatives of the Electrostatic Potential}

The $\mathbf{r} \cdot \boldsymbol{\nabla}$ operator which appears in eq (6) is

$$
\mathbf{r} \cdot \boldsymbol{\nabla}=\left(\sigma_{1}^{\prime} \frac{\partial}{\partial x}+\sigma_{2}^{\prime} \frac{\partial}{\partial y}+\sigma_{3}^{\prime} \frac{\partial}{\partial z}\right)
$$

where $\sigma_{i}^{\prime}=(a / 4) \sigma_{1}$. Let us denote a given term in the series (4) by $T=\left(x^{2}+y^{2}+z^{2}\right)^{-1 / 2}=r^{-1}$. We then list the spatial derivatives up to order four:

$$
\begin{aligned}
\frac{\partial T}{\partial x} & =-\frac{x}{r^{3}} ; \\
\frac{\partial^{2} T}{\partial x \partial y} & =\frac{3 x y}{r^{5}}, \frac{\partial^{2} T}{\partial x^{2}}=\frac{3 x^{2}}{r^{3}}-\frac{1}{r^{3}} ; \\
\frac{\partial^{3} T}{\partial x \partial y \partial z} & =\frac{-15 x y z}{r^{7}}, \frac{\partial^{3} T}{\partial x^{2} \partial y}=\frac{-15 x^{2} y}{r^{7}}+\frac{3 y}{r^{5}},
\end{aligned}
$$

$$
\frac{\partial^{3}}{\partial x^{3}}=-\frac{15 x^{3}}{r^{6}}+\frac{9 x}{r^{5}}
$$

$$
\begin{aligned}
\frac{\partial^{4} T}{\partial x^{2} \partial y \partial z} & =\frac{105 x^{2} y z}{r^{9}}-\frac{15 y z}{r^{7}}, \\
\frac{\partial^{4} T}{\partial x^{3} y} & =\frac{105 x^{3} y}{r^{9}}-\frac{45 x y}{r^{7}}, \\
\frac{\partial^{4} T}{\partial x^{2} \partial y^{2}} & =\frac{105 x^{2} y^{2}}{r^{9}}-\frac{15\left(x^{2}+y^{2}\right)}{r^{7}}+\frac{3}{r^{5}}, \\
\frac{\partial^{4} T}{\partial x^{4}} & =\frac{105 x^{4}}{r^{9}}-\frac{90 x^{2}}{r^{7}}+\frac{9}{r^{5}} .
\end{aligned}
$$

\section{References}

[1] Bennett, H. S., Phys. Rev. 169, 729 (1968).

[2] Gourary, B. S., and Adiran, F. J., Phys. Rev. 105, 1180 (1957).

[3] Kojima, T., J. Phys. Soc. Japan 12, 918 (1957).

[4] Wood, R. F., and Joy, H. W., Phys. Rev. 136, A451 (1964).

[5] Ziman, J. M., Principles of the Theory of Solids, p. 37. (Cambridge University Press, Cambridge, England, 1964).

[6] Tosi, M. P., Solid State Physics 16, 1, edited by F. Seitz and D. Turnbull (Academic Press Inc., New York, 1964).

(Paper 72A5-513) 